\title{
BEITRÄGE ZUR THEORIE DER GRUPPEN LINEARER
}

\section{HOMOGENER SUBSTITUTIONEN*}

\author{
VON
}

\section{SCHUR}

In seiner Arbeit Über die vollständig reduciblen Gruppen, die zu einer Gruppe linearer homogener Substitutionen gehören $\dagger$ hat Herr A. LoEWY eine wichtige neue Art der Reduktion einer Gruppe (S) linearer homogener Substitutionen angegeben, die er als die Zerlegung der Gruppe unter Hervorhebung der zu ihr gehörenden auf einander folgenden grössten vollständig reduziblen Gruppen bezeichnet. Diese Zerlegung kann man, wenn die Koeffizienten aller Substitutionen von $(\mathcal{S}$ einem gegebenen Zahlkörper $\Omega$ angehören, auch innerhalb des Körpers $\Omega$ durchführen. Auf diese Weise erhält Herr LoEwY ein System von gewissen zu $\mathfrak{S S}$ gehörenden Gruppen $\mathfrak{A}_{1}, \mathfrak{A}_{2}, \ldots, \mathfrak{A}_{\mu}$ mit Koeffizienten aus dem Körper $\Omega$, die inbezug auf $\Omega$ vollständig reduzibel sind, und er zeigt auch, dass die Gruppen $\mathfrak{A}_{1}, \mathfrak{A}_{2}, \ldots, \mathfrak{A}_{\mu}$, wenn man äquivalente (ähnliche) Gruppen als nicht von einander verschieden ansieht, in der gegebenen Reihenfolge als eindeutig bestimmt zu betrachten sind.

In der vorliegenden Arbeit soll nun eine, wie ich glaube, nicht unwesentliche Ergänzung der LoEwy'schen Resultate mitgeteilt werden. Es wird nämlich gezeigt, dass die Gruppen $\mathfrak{A}_{1}, \mathfrak{A}_{2}, \ldots, \mathfrak{A}_{\mu}$, wenn wieder äquivalente Gruppen als nicht von einander verschieden gelten, von der Wahl des Körpers $\Omega$ gänzlich unabhängig sind; oder genauer : legt man der Betrachtung an Stelle des Körpers $\Omega$ einen anderen Körper $\boldsymbol{\Omega}^{\prime}$ zugrunde, der ebenfalls alle Substitutionskoeffizienten von $\mathbb{S}$ umfasst, und sind $\mathfrak{A}_{1}^{\prime}, \mathfrak{A}_{2}^{\prime}, \ldots, \mathfrak{A}_{\mu^{\prime}}^{\prime}$ die zu $\mathbb{S}$ gehörenden auf einander folgenden grössten vollständig reduziblen Gruppen inbezug auf $\Omega^{\prime}$, so ist $\mu^{\prime}=\mu$, ferner sind $\mathfrak{A}_{\alpha}$ und $\mathfrak{A}_{\alpha}^{\prime}$ äquivalente Gruppen. $\ddagger$

Zur Begründung dieses Resultats bedarf es einer eingehenderen Untersuchung der in einem Körper $\Omega$ irreduziblen Gruppen linearer homogener Substitutionen. In §§ 2-4 stelle ich für diese Gruppen eine Reihe von Sätzen auf, die

* Presented to the Society (Chicago) A pril 17, 1908.

†Transactions of the American Mathematical Sooiety, vol. 6 (1905), p. 504. Vergl. auch L. Stick elberger, Zur Theorie der vollotändig reduciblen Gruppen, die zu einer Gruppe linearer homogener Substitutionen gehören, ibid., vol. 7 (1906), p. 509.

† Für den Fall, daes \& eine zyklische Gruppe ist, die ans den Potenzen einer Substitution besteht, hat diesen Satz bereits Herr J. WIRTH in seiner Dissertation Ubcr die Elementarteiler einer linearen homogenen Substilution (Freiburg i. Br., 1906) bewiesen. 
ganz analog sind den Sätzen, die ich für den speziellen Fall der endlichen Gruppen bereits in meiner Arbeit Arithmetische Untersuchungen über endliche Gruppen linearer Substitutionen* bewiesen habe. Dass diese Satze auch für unendliche Gruppen ihre Geltung behalten, beruht in erster Linie auf dem Umstand, dass jede Gruppe linearer Substitutionen, die inbezug auf einen gegebenen Körper $\Omega$ irreduzibel ist, im Bereich aller Zahlen vollständig reduzibel ist. $\dagger$ Auf diese bemerkenswerte Tatsache hat bereits Herr TABER am Schluss seiner Arbeit Sur les groupes réductibles de transformations lineaires et homogènes $\ddagger$ aufmerksam gemacht. Nimmt man diesen Satz als bekannt an, so lassen sich auch meine anderen Sätze, insbesondere der hier hauptsächlich in Betracht kommende Satz $X$ leichter beweisen. Es schien mir aber von Interesse, die Untersuchung in der Weise durchzurühren, dass sich zugleich ein neuer Beweis für den TABER'schen Satz ergiebt. Während Herr TABER beim Beweis seines Satzes von der Theorie der hyperkomplexen Grössen Gebrauch macht, stützt sich meine Beweisführung auf einen einfachen Hilfssatz (Satz II), der sich leicht direkt begründen lässt.

\section{$\S 1$.}

Unter einer Gruppe linearer Substitutionen (Matrizen) des Grades $g$ verstehe ich im folgenden ein beliebiges endliches oder auch unendliches System (B) von linearen homogenen Substitutionen in $g$ Variabeln, wenn das Produkt von je zwei Substitutionen von $\mathbb{B}$ wieder in $\mathbb{B}$ enthalten ist. Sind die Koeffizienten aller Substitutionen von (S) Zahlen eines gegebenen Zahlkörpers $\Omega$, so sage ich kurz, $\mathbb{G}$ sei eine in $\Omega$ rationale Gruppe. Ebenso verstehe ich unter einer in $\Omega$ rationalen Matrix eine Matrix, deren Koeffizienten $\operatorname{dem}$ Körper $\boldsymbol{\Omega}$ angehören. Geht nun eine in $\Omega$ rationale Gruppe $\mathbb{B}$ durch die lineare Transformation $T$ der Variabeln in die ihr äquivalente Gruppe $\mathbb{S}^{\prime} \perp T \& T^{-1}$ über, und weiss man, dass (S' $^{\prime}$ wieder in $\Omega$ rational ist, so kann man offenbar $T$ auch so wählen, dass die Koeffizienten von $T$ ebenfalls in $\Omega$ enthalten sind.

Es seien nun $(\mathcal{S}$ und $\mathfrak{S}$ zwei Gruppen linearer Substitutionen der Grade $g$ und $h$, die entweder einander isomorph oder allgemeiner einer dritten Gruppe homomorph $\S$ sind. Lässt sich dann eine Matrix $P$ mit $g$ Zeilen und $h$ Kolonnen oder auch eine Matrix $P$ mit $h$ Zeilen und $g$ Kolonnen bestimmen, sodass für je zwei entsprechende Substitutionen (Matrizen) $G$ und $H$ der beiden Gruppen $\mathfrak{S}$ und $\mathfrak{S}$ die Gleichung $G P=P H$, bezw. die Gleichung $P G=H P$

* Sitzungsberichte der Berliner Akademie, Februar 1906, p. 164. Im folgenden wird diese Arbeit kurz mit $\Lambda$. zitiert.

$\dagger$ Für den Fall, das $\Omega$ den Bereich aller reellen Zahlen bedeutet, hat diesen Satz Herr $\Delta$. LoEWY in seiner Arbeit Über die Reducibilität der reellen Gruppen linearer homogener Subetitutionen, Transactions of the Amerioan Mathematioal Sooiety, vol. 4 (1903), p. 171, bewiesen.

† Comptes Rendus de l'Aoadémie des Soiences, $\triangle$ pril 1906, p. 948.

\$ Vergl. G. Frobrerius und I. SCHUR, Über die Äquivalenz der Gruppen linearer Bubstitutionen, Sitzungoberichte der Berliner Akademie, Februar 1906, p. 209. 
besteht, so will ich $\mathbb{S}$ und $\mathfrak{B}$ als verkettete Gruppen bezeichnen; ich sage auch kürzer, die Matrix $\boldsymbol{P}$ genüge der Gleichung

$$
\mathbb{B P}=\boldsymbol{P G} \quad \text { oder } \quad P \text { B }=\mathfrak{Q} P \text {. }
$$

Natürlich soll hierbei nicht $P=0$ sein. - Sind insbesondere die beiden Gruppen $(\mathcal{S}$ und $\mathfrak{G}$ in einem Körper $\Omega$ rational und weiss man, dass eine von Null verschiedene Matrix $P$ existiert, die einer der Gleichungen (1) genügt, so kann man offenbar auch eine in $\Omega$ rationale Matrix wählen, die dieselbe Bedingung erfüllt. Sind ferner $\mathbb{S}^{\prime}$ und $\mathfrak{S}^{\prime}$ zwei zu $\mathbb{S}$ und $\mathfrak{W}$ äquivalente Gruppen, von denen bekannt ist, dass sie untereinander verkettet sind, so schliesst man leicht, dass auch $\mathbb{B}$ und $\mathfrak{B}$ verkettet sind. Dieser Fall tritt insbesondere ein, wenn die Gruppen $\mathfrak{S}^{\prime}$ und $\mathfrak{G}^{\prime}$ die spezielle Form

$$
\mathbb{G}^{\prime}=\left(\begin{array}{ll}
\mathfrak{A} & 0 \\
\mathfrak{C} & \mathfrak{D}
\end{array}\right), \quad \mathfrak{G}^{\prime}=\left(\begin{array}{cc}
\mathfrak{U} & 0 \\
0 & \mathfrak{C}
\end{array}\right)
$$

aufweisen, wo $\mathfrak{A}$ eine gewisse Gruppe des Grades a bedeuten soll. Denn versteht man alsdann unter $\boldsymbol{P}^{\prime}$ die Matrix

$$
P^{\prime}=\left(p_{k \lambda}\right) \quad(\kappa=1,2, \cdots, n ; \lambda=1,2, \cdots, g),
$$

wo $p_{11}, p_{22}, \cdots, p_{a a}$ gleich 1 , dagegen alle übrigen $p_{k \lambda}$ gleich 0 sind, so wird, wie man leicht findet,

$$
P^{\prime} \mathscr{C}^{\prime}=\mathfrak{G}^{\prime} P^{\prime}=\left(\begin{array}{cc}
\mathfrak{A} & 0 \\
0 & 0
\end{array}\right) \text {. }
$$

Daher sind in diesem Fall $\mathscr{S}^{\prime}$ und $\mathfrak{S}^{\prime}$, und folglich auch $\mathfrak{G}$ und $\mathfrak{E}$ verkettete Gruppen.

Es gilt ferner der für das folgende wichtige Satz:

I. Es seien $\mathbb{8}$ und $\mathfrak{G}$ zwei in einem Körper $\Omega$ rationale Gmuppen linearer Substitutionen der Grade $g$ und $h$, die entweder isomorph oder einer dritten Gruppe homomorph sind, und es sei (S) insbesondere im Körper $\Omega$ irreduzibel. Ist dann $\boldsymbol{P}$ eine in $\Omega$ rationale Matrix, die der Gleichung $\mathbb{S P}=\boldsymbol{P} \mathfrak{G}$ oder der Gleichung $P \mathbb{C}=\mathfrak{P}$ genügt, 80 ist entweder $\boldsymbol{P}=0$ oder es ist $\boldsymbol{P}$ vom Range $g$. Im letzteren Falle enthält $\mathfrak{B}$ die Gruppe $\mathbb{S}$ als irreduziblen Bestandteil inbezug auf $\mathbf{\Omega}$.

Der Beweis dieses Satzes ist genau ebenso zu führen, wie der Beweis des Satzes I meiner mit A. zitierten Arbeit.

Aus dem Satze I ergiebt sich sofort:

I*. Ist (S) eine im Körper $\Omega$ irreduzible Gruppe des Grades $g$ und $\mathfrak{Q}$ eine in $\Omega$ rationale Gruppe des Grades $h$, die mit (S) verkettet ist, 80 muss $h \geqq g$ sein. Ist spexiell $h=g, 80$ muss $\mathfrak{S}$ mit $\mathbb{S}$ äquivalent sein.

Um die Untersuchung später nicht unterbrechen zu müssen, schicke ich noch folgende Bemerkung voraus. 
Es sei

$$
\phi(x)=x^{r}-c_{1} x^{r-1}-c_{2} x^{r-2}-\cdots-c_{r}=0
$$

eine Gleichung mit Koeffizienten aus dem Körper $\boldsymbol{\Omega}$, die in $\boldsymbol{\Omega}$ irreduzibel ist ; die Wurzeln der Gleichung seien $\rho=\rho_{0}, \rho_{1}, \rho_{2}, \cdots, \rho_{r-1}$. Setzt man

und

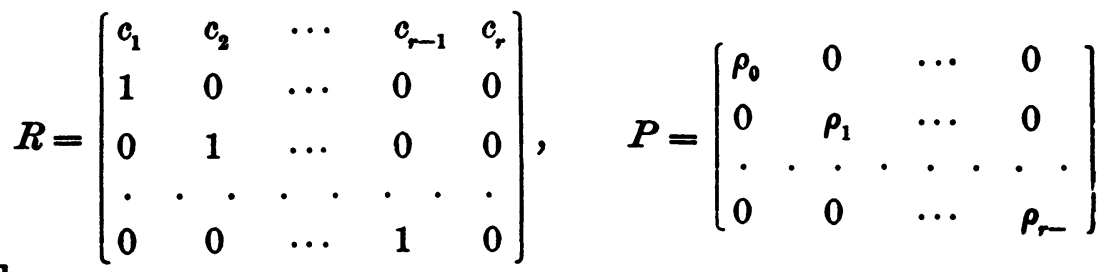

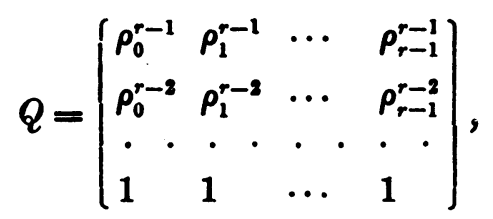

so wird, wie man leicht erkennt,

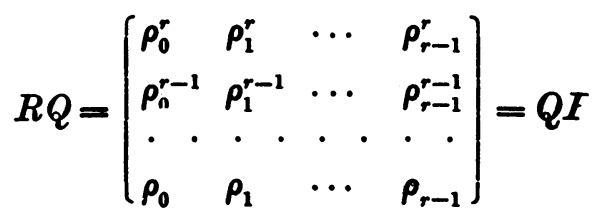

Also ist, da die Determinante der Matrix $Q$ nicht 0 ist,

$$
Q^{-1} R Q=P \text {. }
$$

Hieraus folgt sofort, dass $R$ der Gleichung

$$
R^{r}-c_{1} R^{r-1}-c_{2} R^{r-2}-\cdots-c_{r} E=0
$$

genügt, wo $E$ die Einheitsmatrix bedeutet. - Man habe nun eine Gruppe $\mathfrak{F}$ linearer Substitutionen des Grades $f$, die im Körper $\Omega(\rho)$ rational ist. Es sei

$$
F=\left\{\phi_{k \lambda}(\rho)\right\}
$$

die Koeffizientenmatrix irgend einer Substitution von $\mathfrak{F}$. Ersetzt man in jeder der in $\Omega$ rationalen Funktionen $\phi_{\kappa \lambda}(\rho)$ die Zahl $\rho$ durch die zu $\rho$ konjugierten Zahlen $\rho_{1}, \rho_{2}, \cdots, \rho_{r-1}$, so mögen die (zu $\mathfrak{F}$ isomorphen) Gruppen $\mathfrak{F}_{1}, \mathfrak{F}_{2}, \cdots, \mathfrak{F}_{r-1}$ entstehen. Substituiert man weiter in $\phi_{k \lambda}(\rho)$ für $\rho$ die Matrix $R$, so bilden auch die Matrizen

$$
\bar{F}=\left\{\phi_{k \lambda}(R)\right\},
$$

deren Grad gleich $r f$ ist, eine mit $\mathfrak{F}$ isomorphe Gruppe $\bar{F}$. Dies folgt daraus, dass jede für $\rho$ bestehende Relation mit Koeffizienten aus dem Körper $\Omega$ wegen 
der Gleichung (3) richtig bleibt, wenn die Zahl $\rho$ durch die Matrix $R$ ersetzt wird.* Ist nun

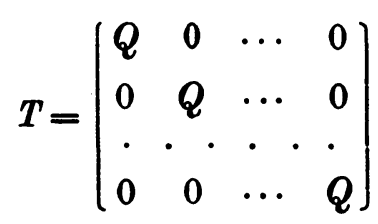

die Matrix des Grades $r f$, die durch $f$-malige Aneinanderreihung der Matrix $Q$ entsteht, so wird $T^{-1} \bar{F} T$, wie aus (2) leicht folgt, eine Gruppe, die durch eine einfache Vertauschung der Zeilen und Spalten in die Gruppe

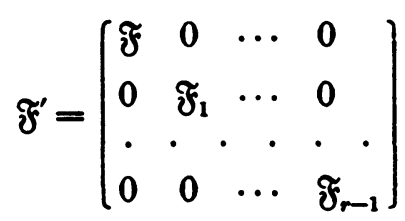

übergeht. Daher ist die Gruppe $\mathfrak{F}^{\prime}$ mit der im Körper $\Omega$ rationalen Gruppe $\bar{F} \ddot{a q u i v a l e n t . \dagger}$

\section{$\S 2$.}

Ist (S) eine im Bereiche $Z$ aller Zahlen irreduzible Gruppe linearer Substitutionen, so hat bekanntlich jede Matrix $\boldsymbol{P}$, die mit allen Substitutionen (Matrizen) von $(B)$ vertauschbar ist, $\ddagger$ die Form $c E$, wo $c$ eine Konstante ist. Weiss man aber umgekehrt, dass jede mit $\mathbb{B}$ vertauschbare Matrix die Form $c E$ hat, so folgt im allgemeinen noch keineswegs, dass \& im Bereiche $Z$ irreduzibel ist. Es gilt jedoch der Satz :

II. Ist \& eine in einern gegebenen Zahlkörper $\Omega$ irreduzible Gruppe und weiss man, dass jede mit \& vertauschbare Matrix die Form $c E$ hat, 80 ist \& auch im Bereiche $\boldsymbol{Z}$ aller Zahlen irreduzibel.

Es sei nämlich \& in $Z$ reduzibel. Dann kann man bekanntlich, wenn $g$ den Grad von 8 bedeutet, jedenfalls $g^{2}$ Konstanten $k_{\alpha \beta}$, die nicht sämmitlich Null sind, bestimmen, sodass für jede Substitution $A=\left(a_{\alpha \beta}\right)$ von $\&$ die Gleichung

$$
\sum_{a, \beta} k_{\beta \alpha} a_{\alpha \beta}=0
$$

besteht. Ich will nun zeigen, dass unter iden über $(\$)$ gemachten Voraussetzungen aus dem Bestehen der Relationen (4) ouch die Reduzibilität von (S) inbezug auf $\Omega$ geschlossen werden kann, was dann auf einen Widerspruch führt.

Der folgende Beweis ist eine fast wörtliche Nachbildung des Beweises, den Herr Frobenius und der Verfasser in der bereits zitierten Arbeit für den

* Vergl. A., p. 172.

† Vergl. L. E. Dicksos, On the reducibility of linear groups, Transaotions of the Amorican Mathomatioal Sooi oty, vol. 4 (1903), p. 434.

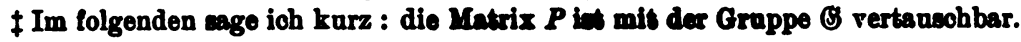


bekannten BuRNsIDE'schen Fundamentalsatz über irreduzible Gruppen angegeben haben. Nur der grösseren Deutlichkeit wegen soll der Beweis hier ausführlich mitgeteilt werden.

Zunächst ist zu beachten, dass die Konstanten $k_{a \beta}$, da sie sich aus linearen Gleichungen mit Koeffizienten aus dem Körper $\Omega$ bestimmen, auch als Zahlen des Körpers $\Omega$ angenommen werden können. Man denke sich nun alle in $\Omega$ rationalen Matrizen $K=\left(k_{\alpha \beta}\right)$ ins Auge gefasst, deren Koeffizienten $k_{\alpha \beta}$ den Gleichungen (4) genügen. Unter ihnen seien höchstens \& linear unabbängig, etwa $K_{1}, K_{2}, \ldots, K_{0}$. Dann lässt sich jede andere solche Matrix $K$ als lineare homogene Verbindung von $K_{1}, K_{2}, \cdots, K_{6}$ mit in $\Omega$ rationalen Koeffizienten darstellen. Nun schliesst man aber sofort, dass auch $K A$ dieselbe Eigenschaft besitzt wie $K$; daher muss sich $K A$ auf die Form $\sum_{\sigma} r_{\sigma} K_{\sigma}$ bringen lassen, wo die $r_{\sigma}$ gewisse Zahlen des Körpers $\Omega$ bedeuten. Speziell sei

$$
K_{\rho} A=\sum_{\sigma=1}^{\dot{r}} r_{\rho \sigma} K_{\sigma} \quad(\rho=1,2, \cdots, s) .
$$

Die Matrizen $R=\left(r_{\rho \sigma}\right)$ bilden dann eine in $\Omega$ rationale Gruppe $\Re$, die mit (S) homomorph ist. Diese Gruppe $\Re$ kann auch inbezug auf $\Omega$ reduzibel sein. In diesem Fall lassen sich $t<8$ linear unabhängige lineare Verbindungen $L_{1}, L_{2}, \cdots, L_{t}$ der $K_{\bullet}$ mit in $\Omega$ rationalen Koeffizienten angeben, sodass

$$
L_{p} A=\sum_{\sigma=1}^{t} \delta_{\rho \sigma} L_{\sigma} \quad(\rho=1,2, \cdots, t)
$$

wird und die (in $\Omega$ rationalen) Matrizen $S=\left(8_{\rho \sigma}\right)$ eine inbezug auf $\Omega$ irreduzible Gruppe $\subseteq$ bilden. - Ist aber $\Re$ in $\Omega$ irreduzibel, so denken wir uns $t=8$ und $S=\Re$ gesetzt. - Bezeichnet man nun, wenn $L_{s}=\left(\chi_{\alpha \beta}\right)$ ist, mit $P_{\alpha}$ die Matrix

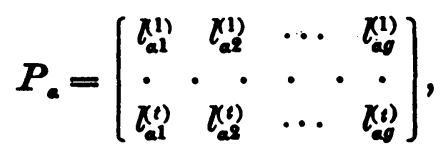

so kann man die Gleichungen (5) auch in der Form

$$
P_{a} A=S P_{a}
$$

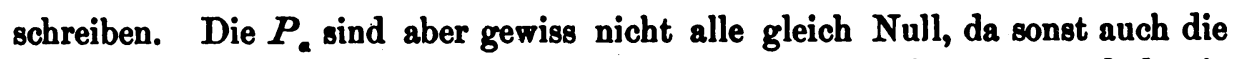
$L_{\rho}$ alle Null wären. Folglich sind $\$(S$ und S verkettete Gruppen, und da sie beide in $\Omega$ irreduzibel sind, so müssen sie nach Satz $I$ einander äquivalent sein. Durch passende Wahl von $L_{1}, L_{2}, \ldots, L_{6}$ kann daher auch erreicht werden, dass $S=A$ wird. Die Gleichungen (6) gehen dann über in die Gleichungen $P_{a} A=A P_{a}$, d. h. $P_{a}$ wird mit $\&$ vertauschbar. Es muss daher auf Grund unserer Annahme über \& die Matrix $P_{a}$ die Form $k_{a} E$ haben; es wird also

$$
\left(x_{\alpha \beta}\right)=k_{\alpha} e_{\gamma \beta} \quad(\alpha, p, \gamma=1,2, \cdots, \theta),
$$


wo $e_{\gamma \beta}$ gleich 1 oder gleich 0 zu setzen ist, je nachdem $\gamma=\beta$ oder $\gamma \neq \beta$ ist. Es soll doch aber

sein ; folglich wird

$$
\sum_{\alpha, \beta}^{(\gamma)} x_{\beta a} a_{\alpha \beta}=0
$$

$$
\sum_{\beta} a_{\gamma \beta} k_{\beta}=0 \text {. }
$$

Wären nun die Konstanten $k_{\beta}$, wie das unsere Annahme, dass die $L_{\gamma}$ linear unabhängig sein sollen, erfordert, nicht sämtlich 0 , so würden diese Gleichungen besagen, dass die Gruppe $(5)$ mit der ihr homomorphen Gruppe, die aus lauter Nullen besteht, verkettet ist. Dies ist aber nach Satz I nicht möglich.

Allgemein gilt der Satz :

III. Ist \$S eine im Körper $\Omega$ irreduzible Gruppe des Grades $g$ und $P$ eine mit (S) vertauschbare Matrix, deren Koeffizienten dem Körper $\Omega$ angehörem, 80 muss die charakteristische Determinante $|x E-P|$ der Matrix $P$ Potenz ciner in $\Omega$ irreduziblen Funktion. $\phi(x)$ sein und es besteht die Gleichung $\phi(P)=0$.

Dieser Satz ist dem Satz II meiner mit A. zitierten Arbeit völlig analog und ist ebenso wie dort zu beweisen.

Nun ist die Bedingung dafür, dass die Matrix $P=\left(p_{a \beta}\right)$ mit der Gruppe $\mathbb{S}$ vertauschbar sein soll, identisch mit einer Reihe von linearen homogenen Gleichungen für die $p_{\alpha \beta}$ mit Koeffizienten aus dem Körper $\boldsymbol{\Omega}$. Denkt man sich daher die allgemeinste mit $\&$ vertauschbare Matrix $P$ bestimmt, so erscheinen die $p_{\alpha \beta}$ als lineare homogene Verbindungen von gewissen 8 Parametern $v_{1}, v_{2}$, $\ldots, v$, mit in $\Omega$ rationalen Koeffizienten. Sind nun unter den charakteristischen Wurzeln dieser allgemeinsten Matrix $\boldsymbol{P}$ genau $\boldsymbol{r}$ unter einander verschieden, so lassen nach einem bekannten Satz der Algebra für die Parameter $v_{a}$ auch spezielle ratio $n$ a $l$ e Werte einsetzen, so dass die 80 entstehende spezielle Matrix $P$ ebenfalls genau $r$ verschiedene charakteristische Wurzeln besitzt. Da aber $P$ alsdann eine in $\Omega$ rationale Matrix wird, so muss nach Satz III

$$
|x E-P|=[\phi(x)]^{\prime}
$$

sein, wo $\phi(x)$ eine in $\Omega$ irreduzible Funktion des Grades $r$ bedeutet und $f=g / r$ zu setzen ist. Ist nun $r=1$, so folgt aus der Gleichung $\phi(P)=0$, dass $P$ die Form $c E$ hat und man schliesst auch sofort, dass die allgemeinste mit \& vertauschbare Matrix ebenfalls diese Form haben muss. In diesem Fall ist daher nach Satz II die Gruppe (S) auch im Bereiche $Z$ aller Zahlen irreduzibel. Ist aber $r>1$ und

$$
\phi(x)=(x-\rho)\left(x-p_{1}\right) \cdots\left(x-p_{r-1}\right),
$$


so folgt aus der Gleichung $\phi(P)=0$, weil die $\rho_{a}$ unter einander verschieden sind, in bekannter Weise, dass die Elementarteiler von $|x E-P|$ sämtlich linear sind.* Hieraus schliesst man leicht, dass sich eine Matrix $R$ von nicht verschwindender Determinante mit Koeffizienten, die dem Körper $\boldsymbol{\Omega}(\rho)$ angehören, bestimmen lässt, so dass

$$
P^{\prime}=R P R^{-1}=\left(\begin{array}{cc}
M & 0 \\
0 & N
\end{array}\right)
$$

wird ; hierin soll $M=\rho E_{f}$ sein, wo $E_{f}$ die Einheitsmatrix des Grades $f$ bedeutet, während unter $N$ eine gewisse in $\Omega(\rho)$ rationale Matrix des Grades $r f-f$ zu verstehen ist, unter deren charakteristischen Wurzeln alsdann nur die Grössen $\rho_{1}, \rho_{2}, \cdots, \rho_{r-1}$ (jede $f \mathrm{Mal}$ ) vorkommen. Man führe nun an Stelle von $\mathbb{E}$ die ihr äquivalente, in $\Omega(\rho)$ rationale Gruppe

$$
\mathbb{S}^{\prime}=R \mathbb{S} R^{-1}
$$

ein. Da nun $\left(\mathcal{S}^{\prime}\right.$ mit der Matrix $P^{\prime}$ vertauschbar ist und die Matrizen $M$ und $N$ keine charakteristische Wurzel gemeinsam haben, so ergiebt sich nach einem bekannten Satz, dass $\mathbb{B}^{\prime}$ die Form

$$
\mathfrak{B S}^{\prime}=\left(\begin{array}{ll}
\mathfrak{F} & 0 \\
0 & \mathfrak{E}
\end{array}\right)
$$

haben muss, wo $\mathfrak{F}$ insbesondere eine Gruppe des Grades $f$ ist. Fs mögen nun $\mathfrak{F}_{1}, \mathfrak{F}_{2}, \cdots, \mathfrak{F}_{r-1}, \overline{\mathfrak{F}}$ und $\mathfrak{F}^{\prime}$ dieselbe Bedeutung haben wie in $\S 1$. Dann sind (vergl. § 1) $\mathbb{S}^{\prime}$ and $\mathfrak{F}^{\prime}$ unter einander verkettete Gruppen. Daher sind auch die ihnen äquivalenten in $\Omega$ rationalen Gruppen $\mathbb{S}$ und $\mathfrak{F}$ verkettet; da aber $\mathbb{B}$ in $\Omega$ irreduzibel und $\bar{F}$ von gleichem Grade $g=r f$ wie $\mathbb{S}$ ist, so müssen $(S)$ und $\bar{F}$ nach Satz I* äquivalente Gruppen sein. Hieraus folgt aber, dis (s) auch der Gruppe

äquivalent ist.

$$
\mathfrak{F}^{\prime}=\left(\begin{array}{cccc}
\mathfrak{F} & 0 & \cdots & 0 \\
0 & \mathfrak{F}_{1} & \cdots & 0 \\
\cdot & \cdot & \cdot & \cdot \\
0 & 0 & \cdots & \mathfrak{F}_{r-1}
\end{array}\right)
$$

Die Gruppe $\mathfrak{F}$ ist jedenfalls im Körper $\Omega(\rho)$ irreduzibel. $\dagger$ Denn wäre dies nicht der Fall, so würde man sofort schliessen können, dass die aus $\mathfrak{F}$ hervorgehende in $\Omega$ rationale Gruppe $\overline{\mathfrak{F}}$ inbezug auf $\Omega$ reduzibel ist; dies ist aber nicht möglich, da $\overline{\mathfrak{F}}$ mit $\mathbb{B}$ äquivalent ist. Es ist aber $\mathfrak{F}$ auch im Körper $Z$ aller Zahlen irreduzibel. Denn wäre die im Körper $\Omega(\rho)$ irreduzible Gruppe $\mathfrak{F}$ in $Z$ reduzibel, so müsste sich nach dem Vorhergehenden eine mit $\mathfrak{F}$ vertauschbare Matrix $L$ angeben lassen, die mindestens zwei verschiedene charak-

* Vergl. A., p. 172.

† Vergl. L. E. Drcrsos, 100. oit. 
teristische Wurzeln $w$ und $w^{\prime}$ besitzt. Versteht man dann unter $w_{1}, w_{2}, \ldots, w_{r-1}$ irgend welche $r-1$ Grössen, die unter einander und auch von $w$ und $w^{\prime}$ verschieden sind, und setzt man

$$
L_{1}=w_{1} E_{f}, L_{2}=w_{2} E_{f}, \cdots, L_{r-1}=w_{r-1} E_{f},
$$

so wird die Matrix

$$
P_{1}=\left(\begin{array}{cccc}
L & 0 & \cdots & 0 \\
0 & L_{1} & \cdots & 0 \\
\cdot & \cdot & \cdot & \cdot \\
0 & 0 & \cdots & L_{r-1}
\end{array}\right)
$$

mit der Gruppe $\mathfrak{F}^{\prime}$ vertauschbar. Eine zu $P_{1}$ ähnliche Matrix $P$ wäre dann mit der Gruppe (S) vertauschbar. Diese Matrix würde jedoch ebenso wie $P_{1}$ mindestens $r+1$ verschiedene charakteristische Wurzeln $w, w^{\prime}, w_{1}, \ldots, w_{r-1}$ besitzen. Dies widerspricht aber der Annahme, die über die Zahl $r$ gemacht worden ist. - Ebenso zeigt man, dass auch die Gruppen $\mathfrak{F}_{1}, \mathfrak{F}_{2}, \ldots, \mathfrak{F}_{r-1}$ im Bereiche $Z$ irreduzibel sind.

Wir erhalten den Satz :

IV. Jede in einem Körper $\Omega$ irreduzible Gruppe (S) linearer Substitutionen ist im Bereich aller Zahlen vollständig reduzibel. Hat die allgemeinste mit (3) vertauschbare Matrix genau $r$ verschiedene charakteristische Wurzeln, so zerfällt (5) im Bereich aller Zahlen in $r$ irreduzible Gruppen desselben Grades. Diese $r$ Gruppen lassen sich auch in $r$ konjugierten algebraischen Körpern über $\Omega$ rational darstellen.

Ferner gilt der Satz :

V. Zwei in einem Körper $\Omega$ irreduzible Gruppen (S) und (i⿱宀 ${ }_{1}$, die entweder isomorph oder einer dritten Gruppe homomorph sind, sind dann und nur dann äquivalent, wenn sie im Bereiche $Z$ aller Zahlen einen irreduziblen Bestandteil gemeinsam haben.

Denn enthalten $\mathbb{S}^{S}$ und $\mathfrak{B S}_{1}$ in $Z$ einen irreduziblen Bestandteil gemeinsam, so sind sie als vollständig reduzible Gruppen unter einander verkettet und folglich nach Satz I" auch äquivalent.

$\S 4$.

Die zu der Gruppe (S) gehörenden im Bereiche $Z$ irreduziblen Gruppen $\mathfrak{F}, \mathfrak{F}_{1}, \cdots, \mathfrak{F}_{r-1}$ brauchen keineswegs in dem Sinne von einander verschieden zu sein, dass nicht zwei von ihnen einander äquivalent sind. Es gilt aber der Satz:

VI. Es möge die im Körper $\Omega$ irreduzible Gruppe (s) im Bereiche $Z$ aller Zahlen in die irreduziblen Gruppen $\mathfrak{F}, \mathfrak{F}_{1}, \ldots, \mathfrak{F}_{r-1}$ zerfallen. Sind dann unter diesen Gruppen genau $l$ vorhanden, von denen nicht zwei einander äquivalent sind, so ist $r / l=m$ eine ganze Zahl und es sind unter den Gruppen $\mathfrak{F}, \mathfrak{F}_{1}, \cdots, \mathfrak{F}_{r-1}$ je $m$ einander äquivalent. 
Dies ergiebt sich fast unmittelbar aus dem Satz, der besagt, dass zwei in $Z$ irreduzible einander isomorphe Gruppen dann und nur dann einander äquivalent sind, wenn je zwei einander entsprechende Substitutionen der beiden Gruppen dieselbe Spur besitzen. * Es mögen nämlich die Spuren der Substitutionen der Gruppe $\mathfrak{F}$ zusammen mit den Zahlen von $\Omega$ einen Körper $\Omega^{\prime}$ erzeugen. Da nun $\mathfrak{F}$ im Körper $\Omega(\rho)$ rational darstellbar ist, so ist $\boldsymbol{\Omega}^{\prime}$ jedenfalls ein Teilkörper von $\Omega(\rho)$ und daher bekanntlich selbst ein algebraischer Körper über $\Omega$. Es möge etwa $\Omega^{\prime}$ aus $\Omega$ durch Adjunktion der Grösse $\chi=\chi(\rho)$ von. $\boldsymbol{\Omega}(\rho)$ hervorgehen, so dass also $\Omega^{\prime}=\Omega(\chi)$ wird. Dann erzeugen die Spuren der Substitutionen der Gruppe $\mathfrak{F}_{\lambda}$, die aus $\mathfrak{F}$ durch die Permutation $\rho \mid \rho_{\lambda}$ hervorgeht, zusammen mit den Zahlen von $\Omega$ den durch die Zahl $\chi_{\lambda}=\chi\left(\rho_{\lambda}\right)$ bestimmten Körper $\Omega\left(\chi_{\star}\right)$. Auf Grund des erwähnten Satzes über die Äquivalenz zweier irreduzibler Gruppen schliesst man sofort, dass $\mathfrak{F}_{k}$ und $\mathfrak{F}_{\lambda}$ dann und nur dànn äquivalente Gruppen sind, wenn $\chi_{\kappa}=\chi_{\Lambda}$ wird. Genügt aber $\chi$ im Körper $\Omega$ einer irreduziblen Gleichung des Grades $l$, so ist bekanntlich $l$ ein Divisor des Grades $r$ des algebraischen Körpers $\Omega(\rho)$ über $\Omega$ und es sind unter den Zahlen $\chi, \chi_{1}, \cdots, \chi_{r-1}$ genau $l$ von einander verschieden und je $m=r / l$ einander gleich. Hieraus folgt aber unser Satz.

Man beweist ferner ganz ähnlich wie bei der analogen Betrachtung A., p. 173 :

VII. Lässt sich die Gruppe F in einem algebraischen Körper $\Omega(\sigma)$ des Grades 8 über $\Omega$ rutional darstellen, so muss \& durch $r$ teilbar sein.

Der früher bestimmte Körper $\Omega(\rho)$ ist also ein algebraischer Körper kleinsten Grades über $\Omega$, in dem die Gruppe $\mathfrak{F}$ rational darstellbar ist.

Ehenso wie für endliche Gruppen (vergl. A., p. 171) gilt auch allgemein der Satz :

VIII. Die Zahl $m=r / l$ ist ein Divisor des Grades $f$ der Gruppe $\mathfrak{F}$.

Der Beweis lässt sich mit Hilfe einer allgemeinen Satzes führen, der auch an und für sich von Wichtigkeit ist :

IX. Man habe eine Gruppe $\mathfrak{Q}$ linearer Substitutionen des Grades $h$, die im Bereiche $Z$ aller Zahlen vollständig reduzibel ist. Unter den irreduziblen Bestandteilen von $\mathfrak{S}$ mögen genau $l$ vorhanden sein, von denen nicht zwei einander äquivalent sind, etwa die Gruppen $\mathfrak{F}_{0}, \mathfrak{F}_{1}, \ldots, \mathfrak{F}_{l-1}$; hierbei soll keine dieser Gruppen aus lauter Nullen bestehen. Ist dann $f_{\lambda}$ der Grad der Gruppe $\mathfrak{F}_{\lambda}$, so sind unter den Substitutionen von $\mathfrak{Q}$ genau

$$
n=f_{0}^{2}+f_{1}^{2}+\cdots+f_{l-1}^{2}
$$

linear unabhängig. Genügen etwa die Substitutionen $H_{1}, H_{2}, \ldots, H_{n}$ von $\mathfrak{Q}$ dieser Bedingung, so sei $H=z_{1} H_{1}+z_{2} H_{2}+\cdots+z_{n} H_{n}$ die allgemeine Substitution von $\mathfrak{G}$ und speziell sei

* G. Frobenius und I. Schor, loc. cit., Satz II. 


$$
H_{a} H_{\beta}=\sum_{\gamma=1}^{n} a_{\gamma \alpha \beta} H_{\gamma}
$$

Bexeichnet man dann mit $S_{a}$ die Matrix

$$
S_{a}=\left(a_{\text {kad }}\right) \quad(\kappa, \lambda=1,2, \cdots, n),
$$

so bilden die Matrizen $S=z_{1} S_{1}+z_{2} S_{2}+\cdots+z_{n} S_{n}$ eine der Gruppe $\mathfrak{G}$ isomorphe Gruppe $S$, die ebenfalls vollständig reduzibel ist. Unter den irreduziblen Bestandteilen von $\subseteq$ kommen wieder nur die l Gruppen $\mathfrak{F}_{0}, \mathfrak{F}_{1}, \cdots, \mathfrak{F}_{t-1}$ vor, und zwor enthält $\subseteq$ die Gruppe $\mathfrak{F}_{\lambda}$ genau $f_{\lambda} M a l$.

Auf die Tatsache, dass die Gruppe $\mathfrak{S}$ zugleich mit der Gruppe $\mathfrak{G}$ vollständig reduzibel ist, hat bereits Herr TABER in seiner in der Einleitung zitierten Arbeit aufmerksam gemacht. Was hier also hinzugefügt wird, ist nur die Bestimmung der irreduziblen Bestandteile von $\mathbb{S}$. Ich bemerke noch, dass unser Satz für den besonderen Fall, dass die Gruppe $\mathfrak{K}$ irreduzibel ist, bereits von Herrn Burnside.* bewiesen worden ist.

Der Beweis des Satzes IX ergiebt sich folgendermassen.

Es möge der Substitution $H_{a}$ von $\mathfrak{W}$ in $\mathfrak{F}_{\lambda}$ die Substitution $F_{a}^{(\lambda)}$ entsprechen. Sind dann $x_{1}, x_{2}, \cdots, x_{n}$ unabhängige Variable und setzt man

$$
X^{(\lambda)}=x_{1} F_{1}^{(\lambda)}+x_{2} F_{2}^{(\lambda)}+\cdots+x_{n} F_{n}^{(\lambda)}
$$

sa sind, weil unter den irreduziblen Gruppen $\mathfrak{F}_{0}, \mathfrak{F}_{1}, \ldots, \mathfrak{F}_{l-1}$ nicht zwei einander äquivalent sind, die $f_{0}^{2}+f_{1}^{2}+\cdots+f_{l-1}^{2}$ Koeffizienten der $l$ Matrizen $X^{(0)}, X^{(1)}, \ldots, X^{(l-1)}$, als Funktionen der $x_{v}$ betrachtet, linear unabhängig. $\dagger$ Andererseits sind unter den $h^{2}$ Koeffizienten der Matrix

$$
x_{1} H_{1}+x_{2} H_{2}+\cdots+x_{n} H_{n}
$$

genau $n$ linear unabhängig. Hieraus schliesst man wegen der über $\mathfrak{G}$ gemachten Annahmen sofort, dass $n=f_{0}^{2}+f_{1}^{2}+\cdots+f_{l-1}^{2}$ sein muss. Man erkennt auch leicht, dass das durch die Gleichungen (7) definierte System hyperkomplexer Grössen nach der von Herrn Frobenius $\ddagger$ eingeführten Bezeichnungsweise ein DEDEKIND'scher System ist, $d$. h. es muss die Determinante $D$ der $n^{2}$ Grössen

$$
p_{\alpha \beta}=\sum_{\alpha, \lambda} a_{\kappa \lambda \alpha} a_{\lambda \alpha \beta} \quad(\alpha, \beta=1,2, \cdots, n)
$$

* On the arithmetical nature of the coefficients in a group of linear substitutions of finite order (second paper), Proceedings of the London Mathematioal Society, ser. 2, vol. 4 (1906), p. 1.

† Vergl. G. Frobenius und I. SoHUR, loo. cit., Sate I.

† Theorie der hyperkomplexen Grözsen, Sitzungsberiohte der Berliner Akademie, 1903, p. 504 und p. 634. 
von Null verschieden sein.* Daher ist die durch die Matrizen

$$
x_{1} S_{1}+x_{2} S_{2}+\cdots+x_{n} S_{n}
$$

gebildete Gruppe $\overline{\mathfrak{S}}$ eine vollständig reduzible Gruppe, die jeden ihrer irreduziblen Bestandteile genau so oft enthält wie sein Grad angiebt. $\dagger$ Dasselbe gilt natürlich auch für die mit $\mathfrak{W}$ isomorphe Gruppe $\mathfrak{S}$. Nun ist aber auch

Setzt man

$$
F_{a}^{(\lambda)} F_{\beta}^{(\lambda)}=\sum_{\gamma} a_{\gamma \alpha \beta} F_{\gamma}^{(\lambda)}
$$

$$
F_{a}^{(\lambda)}=\left(k_{\mu \nu}^{(a)}\right)
$$$$
\left(\mu, \nu=1,2, \cdots, f_{\lambda}\right)
$$

und bezeichnet mit $P_{v}$ die Matrix

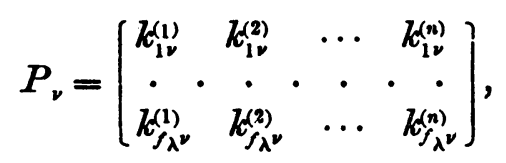

so lassen sich die Gleichungen (8) auch in der Form

$$
F_{a}^{(\lambda)} P_{v}=P_{v} S_{a}
$$

schreiben. Da nun die $P_{v}$ nicht sämtlich 0 sein können, so besagen diese Gleichungen (vergl. $\S 1$ ), dass die Gruppe $\subseteq$ mit der Gruppe $\mathfrak{F}_{\lambda}$ verkettet ist. Nach Satz I muss daher $\mathfrak{F}_{\lambda}$ ein irreduzibler Bestandteil von $\mathfrak{S}$ sein und ist nach dem Gesagten genau $f_{\lambda} \mathrm{Mal}$ in $\subseteq$ enthalten. $\ddagger \mathrm{Da}$ dies für jedes $\lambda$ gilt, so erhält man $f_{0}+f_{1}+\cdots+f_{l-1}$ irreduzible Bestandteile von $\mathfrak{S}$, die aneinander gereiht eine Gruppe des Grades $f_{0}^{2}+f_{1}^{2}+\cdots+f_{l-1}^{2}=n$ ergeben. Da aber $n$ der Grad von $\subseteq$ ist, so kann $\subseteq$ keinen weiteren irreduziblen Bestandteil enthalten. §

Ist nun speziell $\mathfrak{Q}=\mathscr{G}$, wie früher, eine im Körper $\Omega$ irreduzible Gruppe, so wird $f_{0}=f_{1}=\cdots=f_{l-1}=f$, also $n=l f^{2}$. Ferner gehören die Zahlen $a_{\gamma a \beta}$ in diesem Fall ebenfalls den Körper $\Omega$ an. Denkt man sich nun die in $\Omega$ ratio-

* Vergl. TABER, 100. cit. - Herr TABEr hat noch die wiohtige Bemerkung hinzugefügt, daes

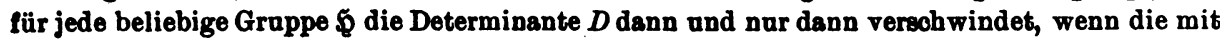
Hilfe der Spuren $q_{a \beta}$ der Matrizen $H_{\alpha} H_{\beta}$ gebildete Determinante $\Delta=\left|q_{a \beta}\right|$ gleich Null ist. Do lässt sich anch, wie bei dieser Gelegenheit hervorgehoben sei, zeigen, dass die Determinanten $D$ und $\Delta$ stets denselben Rang besitzen.

† Dass die zu einem System hyperkomplexer Grössen mit nicht versoh windender Determinante $D$ gehörende Gruppe $\tilde{S}$ diese Eigenschaft besitzt, bat zuerst Herr MoLIEN in seiner Arbeit ther Systeme höherer complexer Zahlen, Mathematische Annalen, Bd. 41 (1893), p. 83, gezeigt. Vergl. anch CARTAN, Sur les groupes bilinéaires et les systèmes de nombres complexes, Annales de Toulouse, t. XII (1898), p. 1 und Frobenius, loo. cit.

‡ Vergl. anch Frobenios, loc. cit., p. 536.

§ Erzeugen die Spuren der Substitutionen der (vollständig reduziblen) Gruppe $\mathfrak{G}$ den Ratioralitätsbereich $\Omega^{\prime}, 80$ gehören auch die Zahlen $a_{\text {yaß }}$ dem Körper $\Omega^{\prime}$ an (vergl. BuRNsIdE, loc. oit., p. 4). Auf Grund dieser Bemerkung kann man weiter sohliessen, dass die Gruppe $\mathfrak{S}$ in einem algebraischen Körper über $\Omega^{\prime}$ rational darstellbar iat. 
nale Gruppe $\mathbb{S}$ inbezug auf $\Omega$ in irreduzible Bestandteile $\mathbb{G}_{1}, \mathbb{S}_{2}, \cdots, \mathbb{S}_{p}$ zerlegt, so muss jede dieser Gruppen der Gruppe (B) äquivalent sein. Denn wäre dies für die Gruppe $\mathbb{B}_{\lambda}$ nicht der Fall, so wären nach Satz V die irreduziblen Bestandteile von $\mathbb{S}_{\lambda}$ inbezug auf den Körper $Z$ aller Zahlen von denjenigen der Gruppe (S) wesentlich verschieden, folglich müsste auch die Gruppe S, im Körper $Z$ zerlegt, einen irreduziblen Bestandteil enhalten, der keiner der Gruppen $\mathfrak{F}, \mathfrak{F}_{1}, \cdots, \mathfrak{F}_{r-1}$ äquivalent ist. Dies widerspricht aber dem soeben Bewiesenen. Daher ist

$$
n=l f^{2}=p g=p m l f,
$$

und hieraus folgt, dass die Zahl $m$ in der Tat ein Divisor der Zahl $f$ ist.

\section{$\S 5$.}

Es sei nun $\Omega$ eine beliebige im Körper $\Omega$ rationale Gruppe linearer Substitutionen des Grades $k$. Diese Gruppe denken wir uns zunächst im Körper $\Omega$ in irreduzible Bestandteile zerlegt, es sei etwa

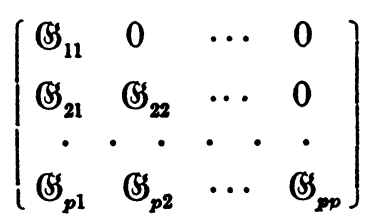

ein in $\Omega$ rationale, mit $\Omega$ äquivalente Gruppe, wo $\mathbb{S}_{11}, \mathbb{S}_{22}, \ldots, \dot{S}_{p p}$ in $\Omega$ irreduzibel sind. Es möge ferner die Gruppe $\mathbb{S}_{a a}$, im Bereiche $Z$ aller Zahlen zerlegt, die irreduziblen Bestandteile $\mathfrak{F}_{a 1}, \mathfrak{F}_{a 2}, \cdots, \mathfrak{F}_{a r_{a}}$ aufweisen. Dann repräsentieren die sich so ergebenden $r_{1}+r_{2}+\cdots+r_{p}$ Gruppen $\mathfrak{F}_{a \beta}$ in ihrer Gesamtheit die irreduziblen Bestandteile der Gruppe $\mathcal{S}$. Auf Grund der früher gewonnenen Resultate kann man nun den Satz aussprechen :

X. Man habe eine Gruppe $\&$ linearer homogener Substitutionen, die in einem Zahllörper $\Omega$ rational ist. Ist dann $\mathfrak{F}$ einer der irreduziblen Bestandteile, in die $\mathfrak{R}$ im Bereiche aller Zahlen zerfällt, so erzeugen die Spuren der Substitutionen von $\mathfrak{F}$ zusammen mit den $Z$ ahlen von $\Omega$ einen algebraischen $K \ddot{K}$ oper über $\Omega$, ferner lässt sich $\mathfrak{F}$ in einem algebraischen $K$ örper $\Omega(\rho)$ über $\Omega$ rational darstellen. Wählt man den Grad $r$ des Körpers $\Omega(\rho)$ möglichst klein, so seien $\rho_{1}, \rho_{2}, \ldots, \rho_{r-1}$ die zu $\rho$ konjugierten Zahlen und es möge $\mathfrak{F}$ in $\mathfrak{F}_{\lambda}$ übergehen, wenn in allen Substitutionskoeffizienten von $\mathfrak{F}$ die Zahl $\rho$ durch $\rho_{\lambda}$ ersetzt wird. Dann ist die Gruppe

$$
\mathfrak{F}^{\prime}=\left(\begin{array}{cccc}
\mathfrak{F} & 0 & \cdots & 0 \\
0 & \mathfrak{F}_{1} & \cdots & 0 \\
\cdot & \cdot & \cdot & \cdot \\
0 & 0 & \cdots & \mathfrak{F}_{r-1}
\end{array}\right)
$$


einer in $\Omega$ rationalen und irreduziblen Gruppe $\mathbb{B}$ äquivalent, die in $\Omega$ als irreduzibler Bestandteil inbexug auf $\Omega$ enthalten ist.

Ich stelle nun folgende Betrachtung an. Es sei

$$
\bar{x}_{\kappa}=\sum_{\lambda=1}^{k} c_{\kappa \lambda} x_{\lambda}
$$

eine beliebige Substitution von $\Omega$. Ist $\phi\left(x_{1}, x_{2}, \ldots, x_{n}\right)$ irgend eine homogene Form der Variabeln $x_{1}, x_{2}, \ldots, x_{n}$, so möge

$$
\phi\left(\bar{x}_{1}, \bar{x}_{2}, \ldots, \bar{x}_{n}\right)=\bar{\phi}\left(x_{1}, x_{2}, \cdots, x_{n}\right)
$$

gesetzt werden. Man denke sich nun eine der Gruppe $\Omega$ homomorphe Gruppe $\mathfrak{A}$ des Grades $a$ gegeben, in der der Substitution $K$ von $\Omega$ die Substitution $A=\left(a_{\alpha \beta}\right)$ entsprechen möge. Es kann dann eintreten, dass sich gewisse $a$, nicht notwendig linear unabhängige, Formen $\phi_{1}, \phi_{2}, \ldots, \phi_{a}$ gleicher Ordnung angeben lassen, so dass

$$
\bar{\phi}_{\alpha}=\sum_{\beta=1}^{a} a_{\alpha \beta} \phi_{\beta}
$$

wird. Ist dies für jede Substitution $K$ von $\Omega$ der Fall, so will ich sagen : $\phi_{1}, \phi_{2}, \cdots, \phi_{a}$ bilden ein zur Gruppe $\mathfrak{A}$ gehörendes Invariantensystem von $\mathfrak{A}$. Sind insbesondere die Formen $\phi_{1}, \phi_{2}, \cdots, \phi_{a}$ unter einander linear unabhängig, und weiss man, dass für jede Substitution $K$ von $\Omega$ Gleichungen der Form (9) bestehen, so sind die Koeffizienten $a_{\alpha \beta}$ eindeutig bestimmt. Die Substitutionen $A=\left(a_{\alpha \beta}\right)$ bilden dann von selbst eine der Gruppe $\boldsymbol{\Omega}$ homomorphe Gruppe. In diesem Falle sage ich auch : $\phi_{1}, \phi_{2}, \ldots, \phi_{a}$ bilden ein Invariantensystem von $\Re$ mit der Transformationsgruppe $\mathfrak{A}$. Für die Gleichungen (9) schreibe ich in jedem Falle auch kürzer

$$
\left(\bar{\phi}_{a}\right)=\mathfrak{A}\left(\phi_{a}\right) .
$$

Es gilt nun der für viele Anwendungen nützliche Satz :

XI. Es sei $\mathfrak{A}$ eine im Körper $\Omega$ irreduzible Gruppe, die der in $\Omega$ rationalen Gruppe $\Omega$ homomorph ist. Hat man dann ein zur Gruppe $\mathfrak{A}$ gehörendes Invariantensystem $\phi_{1}, \phi_{2}, \cdots, \phi_{a}$ von $\Omega$ mit Koeffizienten aus dem Körper $\Omega$, so sind die Formen $\phi_{1}, \phi_{2}, \cdots, \phi_{a}$ entweder alle Null oder sie sind unter einander linear unabhängig.

Dies ist leicht zu beweisen. Denn es seien die Formen $\phi_{a}$ nicht alle Null ; man bezeichne die Anzahl der linear unabhängigen unter ihnen mit $r$. Dann lassen sich also $r$ linear unabłängige Formen $\psi_{1}, \psi_{2}, \cdots, \psi_{r}$ mit Koeffizienten aus dem Körper $\Omega$ angeben, so dass Gleichungen der Form

$$
\psi_{p}=\sum_{a=1}^{a} p_{\rho a} \phi_{a}, \quad \phi_{a}=\sum_{p=1}^{r} q_{a p} \psi_{p}
$$


bestehen, wo die $p_{\rho a}$ und $q_{a p}$ Zahlen von $\Omega$ sind. Aus (10) folgt, wenn

gesetzt wird,

$$
r_{\rho \sigma}=\sum_{a, \beta=1}^{a} p_{\rho a} a_{\alpha \beta} q_{\beta \sigma}
$$

$$
\bar{\psi}_{\rho}=\sum_{\sigma=1}^{r} r_{\rho \sigma} \psi_{\sigma} .
$$

Bezeichnet man daher die durch die Substitutionen $R=\left(r_{\rho \sigma}\right)$ gebildete Gruppe mit $\Re$, so erscheinen die Formen $\psi_{1}, \psi_{2}, \ldots, \psi_{r}$ als ein Invariantensystem von $\mathcal{A}$ mit der Transformationsgruppe $\mathfrak{R}$. Andererseits wird aber

also ist

$$
\bar{\phi}_{a}=\sum_{\rho} q_{a \rho} \bar{\psi}_{\rho}=\sum_{\rho, \sigma} q_{a \rho} r_{\rho \sigma} \psi_{\sigma}=\sum_{\beta} a_{a \beta} \phi_{\beta}=\sum_{\beta, \sigma} a_{a \beta} q_{\beta \sigma} \psi_{\sigma},
$$

$$
\sum_{\rho=1}^{r} q_{\alpha \rho} r_{\rho \sigma}=\sum_{\beta=1}^{a} a_{\alpha \beta} q_{\beta \sigma} .
$$

Setzt man die Matrix $\left(q_{a p}\right)$ gleich $Q$, so wird auf Grund der in $\S 1$ festgesetzten Bezeichnungeweise

$$
Q \Re=\mathfrak{A} Q .
$$

Da nun $\mathfrak{A}$ in $\Omega$ irreduzibel ist, so muss nach Satz I der Rang von $Q$ gleich $a$ sein. Dies erfordert aber, dass $r=a$ wird.*

Es sei nun $\mathfrak{F}$ wie früher ein irreduziblen Bestandteil des Grades $f$ von $\Omega$ inbezug auf den Bereich aller Zahlen. Ich nehme insbesondere an, dass sich $f$ (nicht identisch verschwindende) lineare homogene Formen $u_{1}, u_{2}, \ldots, u_{f}$ der $\boldsymbol{x}_{\kappa}$ angeben lassen, die ein zu $\mathfrak{F}$ gehörendes Invariantensystem von $\mathfrak{A}$ bilden; es sei auch noch verlangt, dass $u_{1}, \cdots, u$, von gewissen gegebenen $a$ linearen homogenen Formen $y_{1}, y_{2}, \ldots, y_{a}$ mit in $\Omega$ rationalen Koeffizienten linear unabhängig sein sollen. Hat nun $\mathfrak{F}$ diese Eigenschaft, so gilt dies auch für jede mit $\mathfrak{F}$ äquivalente Gruppe. Denkt man sich insbesondere $\mathfrak{F}$ durch eine äquivalente Gruppe ersetzt, die in dem oben genannten Körper $\boldsymbol{\Omega}(\rho)$ rational ist, so lassen sich die Linearformen $u_{1}, u_{2}, \ldots, u_{f}$ auch so wählen, dass ihre Koeffizienten ebenfalls Zahlen von $\Omega(\rho)$ werden, ohne dass die Linearformen auf hören den früher gestellten Forderungen zu genügen. Geht dann $u_{a}=u_{a 0}$ in $u_{a v}$ über, wenn $\rho$ durch $\rho_{\nu}$ ersetzt wird, so bilden die $f$ Linearformen $u_{1 v}$, $u_{2 v}, \cdots, u_{\rho v}$ ein zur Gruppe $\mathfrak{F}_{\nu}$, ferner die $r f=g$ Linearformen $u_{a \nu}$ zusammengenommen ein zur Gruppe $\mathfrak{F}^{\prime}$ gehörendes Invariantensystem von $\mathfrak{\Omega}$.

Setzt man nun w siter

$$
z_{a \mu}=\sum_{\nu=0}^{r-1} \rho_{\nu}^{\mu} u_{a v} \quad(\alpha=1,2, \cdots, f ; \mu=0,1, \cdots, r-1),
$$

* Nimmt man $\mathscr{A}$ nicht als irreduzibel an, so ergiebt sich, dass $\mathfrak{A}$ einer in $\mathbb{Q}$ rationalen Gruppe der Form

äquivalent ist.

$$
\left(\begin{array}{ll}
\Re & \mathbb{Q} \\
0 & \Re
\end{array}\right)
$$


so werden die Koeffizienten dieser $r f=g$ Linearformen der $x_{\kappa}$, als symmetrische Funktionen der $\rho_{\nu}$, Zahlen des Körpers $\Omega$. $^{*}$ Ist ferner $T$ die Koeffizientenmatrix der Formen $z_{a \mu}$, als Funktionen der $u_{a \nu}$ aufgefasst, und setzt man

$$
T \mathfrak{F}^{\prime} T^{-1}=\mathfrak{G},
$$

so erkennt man sofort, dass die $z_{a \mu}$ ein zur Gruppe $(S)$ gehörendes Invariantensystem von $\Re$ bilden. Die Gruppe (S) ist aber (vergl. $\S 1$ ) eine in $\Omega$ rationale und irreduzible Gruppe. Folglich müssen nach Satz XI die Linearformen $z_{a \mu}$, da sie offenbar nicht alle Null sein können. unter einander linear unabhängig sein.

\section{$\S 6$.}

Ich wende mich nun zur Betrachtung des in der Einleitung erwähnten Satzes von Herrn A. LoEwy.

Der Gedankengang des Herrn LNEWY lässt sich folgendermassen formulieren. Man habe eine im Körper $\Omega$ rationale und in diesem Körper vollständig reduzible Gruppe $\mathfrak{A}$ des Grades $a$, die zu unserer Gruppe $\mathfrak{A}$ homomorph ist. Es mögen sich ferner $a$ unabhängige Linearformen $y_{1}, y_{2}, \cdots, y_{a}$ der $x_{\kappa}$ mit Koeffizienten aus den Körper $\Omega$ bestimmen lassen, die ein Iuvariantensystem von $\Omega$ mit der Transformationsgruppe $\mathfrak{A}$ bilden. Wir verlangen weiter, dass die Gruppe $\mathfrak{A}$ noch folgender Bedingung genügen soll : es soll sich keine in $\Omega$ irreduzible mit $\Omega$ homomorphe Gruppe $\mathbb{S}$ des grades $g$ angeben lassen, zu der ein lineares Invariantensystem $z_{1}, z_{2}, \cdots, z_{g}$ von $\Omega$ mit Koeffizienten aus $\Omega$ in der Weise gehört, dass die $a+g$ Linearformen $y_{1}, y_{2}, \cdots, y_{a}, z_{1}, \cdots, z_{g}$ unter einander linear unabhängig werden. Dann beweist Herr LoEwY :

“Ist $\overline{\mathfrak{A}}$ eine zweite Gruppe, welche genau denselben Forderungen genügt, wie die Gruppe $\mathfrak{A}$, so sind $\mathfrak{A}$ und $\overline{\mathfrak{A}}$ äquivalente Gruppen. Bestimmt man ferner, was jedenfalls möglich ist, zwei zu $\Omega$ äquivalente in $\Omega$ rationale Gruppen $\mathfrak{K}^{\prime}$ und $\bar{\AA}$ der Form

$$
\mathfrak{R}^{\prime}=\left(\begin{array}{cc}
\mathfrak{A} & 0 \\
\mathfrak{C} & \mathfrak{D}
\end{array}\right), \quad \overline{\mathfrak{A}}=\left(\begin{array}{cc}
\overline{\mathfrak{A}} & 0 \\
\overline{\mathfrak{C}} & \overline{\mathfrak{D}}
\end{array}\right),
$$

so sind auch $\mathfrak{D}$ und $\bar{D}$ äquivalente Gruppen." $†$

Man kann daher $\mathfrak{A}$ als die grösste zu $\mathfrak{A}$ gehörende vollständig reduzible Gruppe inbezug auf $\Omega$ bezeichnen.

Tritt nun an Stelle des Körpers $\Omega$ ein anderer Körper $\Omega^{\prime}$, der ebenso wie $\Omega$ alle Koeffizienten der Substitutionen von $\mathscr{R}$ umfasst, so existiert auch inbezug auf $\Omega^{\prime}$ eine zu $\mathfrak{R}$ gehörende grösste vollständig reduzible Gruppe $\mathfrak{A}^{\prime}$.

* Vergl. L. E. Dickson, loo. oit.

† Herr Lozwy macht in seiner Arbeit noch die Voraussetzung, dass die Determinanten der Substitutionen von $\Re$ nicht versoh winden, und dass die reziproke Substitution jeder Substitution von $\Omega$ wieder in $\Re$ enthalten ist. Das von Herrn Stickelberarr (vergl. das in der Einleitung angeführte Zitat) mitgeteilte Beweisverfahren gestattet aber, den LorwY'schen Satz auch für eine allgemeine Gruppe $\Re \mathrm{zu}$ beweisen. 
Ich will nun zeigen, dass $\mathfrak{A}$ und $\mathfrak{A}^{\prime}$ äquivalente Gruppen sind.

Es genügt offenbar, dies allein für den Fall zu beweisen, dass $\Omega^{\prime}$ den Bereich $\boldsymbol{Z}$ aller Zahlen bedeutet. Der Beweis gestaltet sich nun folgendermassen.

Da jede im Körper $\Omega$ irreduzible Gruppe im Körper $Z$ vollständig reduzibel ist, so ist die in $\Omega$ vollständig reduzible Gruppe $\mathfrak{A}$ in $Z$ ebenfalls vollständig reduzibel. Wir haben also nur zu zeigen, dass $\mathfrak{A}$ auch, im Körper $Z$ betrachtet, die Eigenschaften einer grössten vollständig reduziblen Gruppe besitzt.

Wäre dies nun nicht der Fall, so müsste sich eine zu $\mathfrak{K}$ homomorphe in $Z$ irreduzible Gruppe $\mathfrak{F}$ des Grades $f$ angeben lassen, zu der ein lineares Invariantensystem $u_{1}, u_{2}, \cdots, u_{f}$ gehört, so dass die $a+f$ Linearformen $y_{1}, \cdots, y_{a}$, $u_{1}, \ldots, u_{f}$ unter einander linear unabhängig sind. Die Gruppe $\mathfrak{F}$ wird dann ein irreduzibler Bestandteil von $\Re$ inbezug auf $Z$. Man bestimme nun, wie im vorigen Paragraphe, die aus $\mathfrak{F}$ hervorgehende inbezug auf $\Omega$ irreduzible Gruppe (8). Dann gehört auch zu dieser, wie wir gesehen haben, ein lineares Invariantensystem $z_{\alpha \mu}$ mit in $\Omega$ rationalen Koeffizienten. Da aber $\mathfrak{A}$ inbezug auf $\Omega$ eine grösste vollständig reduzible Gruppe sein soll, so dürften die Linearformen $z_{a \mu}$ und $y_{1}, y_{2}, \cdots, y_{a}$ nicht unter einander linear unabbängig sein. Hieraus folgt aber (wegen der Irreduzibilität von $\mathbb{S}$ inbezug auf $\Omega$ ) nach einem von Herrn Stickelberger, loc. cit., bewiesenen Satze, dass jede der Funktionen $z_{a \mu}$ durch $y_{1}, y_{2}, \cdots, y_{a}$ linear darstellbar sein muss. Es ist aber, wie aus den Gleichungen (11) hervorgeht, jede der Funktionen $u_{1}, u_{2}, \cdots, u_{f}$ eine lineare homogene Form der $z_{a \mu}$ und wäre folglich auch durch $y_{1}, y_{2}, \ldots, y_{a}$ linear und homogen darstellbar. Dies führt aber auf einen Widerspruch.

Ist wie früher

$$
\mathfrak{R}^{\prime}=\left(\begin{array}{cc}
\mathfrak{A} & 0 \\
\mathfrak{C} & \mathfrak{D}
\end{array}\right)
$$

eine zu $\Omega$ äquivalente in $\Omega$ rationale Gruppe, so gehört auch zur Gruppe $D$ inbezug auf $\Omega$ eine grösste vollständig reduzible Gruppe u. s. f. So erhält Herr LoEwy die zu $\Re$ inbezug auf $\Omega$ gehörenden auf einander folgenden grössten vollständig reduziblen Gruppen $\mathfrak{A}_{1} \mathfrak{A}_{1}, \ldots, \mathfrak{A}_{\mu}$. Aus unserer Betrachtung ergiebt sich aber, dass diese Gruppen in dem in der Einleitung genauer erläuterten Sinne von der Wahl des Körpers $\Omega$ unabhängig sind.

Man kann dieses Resultat auch folgendermassen aussprechen :

XII. Ist $\Re$ eine in einem gegebenen $Z$ ahlkörper $\Omega$ rationale Gruppe linearer homogener Substitutionen und zerlegt man $\mathfrak{\Re}$ im Bereich aller Zahlen unter Hervorhebung der zu $\mathfrak{i}$ gehörenden auf einander folgenden grössten vollstïndig reduziblen Gruppen, so ist jede dieser Gruppen im Körper $\Omega$ rational darstellbar.

BERLIN, im Mărz 1908. 